\title{
Descriptores del Desarrollo de la Mirada Profesional en el Contexto de la Generalización de Patrones
}

\author{
Descriptors of the Professional Look Development in the Context of \\ Pattern Generalization
}

\begin{abstract}
Resumen
El objetivo de esta investigación es generar descriptores del desarrollo de la competencia docente mirar profesionalmente el pensamiento matemático de los alumnos en estudiantes para maestro (EPM), en el contexto de la generalización de patrones. Para ello, se pidió a futuros maestros que describieran las respuestas de tres alumnos de Primaria a un problema de generalización de patrones, que interpretaran la comprensión de los alumnos y que propusieran acciones para mejorar o ampliar el proceso de enseñanza-aprendizaje. A partir de las destrezas que conceptualizan la mirada profesional (identificar, interpretar y decidir) se generaron descriptores de cuatro niveles de desarrollo: (1) EPM que no identifican los elementos matemáticos relevantes en el proceso de generalización de patrones, (2) EPM que identifican los elementos matemáticos, pero no los usan para interpretar la comprensión de los alumnos, (3) EPM que identifican los elementos y los usan para interpretar la comprensión de los alumnos y (4) EPM que identifican los elementos, los usan para interpretar la comprensión de los alumnos y proponer acciones adecuadas para el progreso del aprendizaje de los alumnos de Primaria. Los niveles establecidos y sus descriptores pueden ser el punto de partida para establecer una futura trayectoria de aprendizaje de la mirada profesional y ofrecer información a los formadores para interpretar el progreso de los EPM en el desarrollo de la competencia mirar profesionalmente el pensamiento matemático de los alumnos en el contexto de la generalización de patrones.
\end{abstract}

Palabras clave: Mirada profesional. Generalización de patrones. Descriptores del desarrollo. Práctica docente. Resolución de problemas.

\begin{abstract}
The objective of this research is to generate descriptors of the development of the competence of professional looking into children's mathematical thinking for prospective teachers (PPT) in the context of pattern generalization. To this end, future teachers were asked to describe the answers of Elementary education students to one problem of the pattern generalization, interpret the students' understanding and propose actions to improve or enlarge the teaching-learning process. From the skills that conceptualize the professional look (identifying, interpreting, and deciding) descriptors of four development levels were generated: (1) PPT that does not identify the relevant mathematical elements in the process of pattern generalization, (2) PPT that identifies the mathematical elements, but does not use these elements to interpret the students understanding, (3) PPT that identifies the elements and uses these elements to interpret the students' understanding and (4) PPT that identifies the elements, uses them to interpret the students' understanding and proposes appropriate actions for the Elementary education students' learning progress. The generated descriptors and the determined levels can be the starting point in establishing a future learning trajectory of the professional look and offering information to
\end{abstract}

\footnotetext{
* Doctor en Didáctica de la Matemática por la Universidad de Alicante (UA). Profesor adjunto en la Universidad Cardenal Herrera CEU en el Campus de Elche (UCH-CEU), Elche, Alicante, España. Dirección postal: Calle Carmelitas, 3, 03203 Elche (Alicante).E-mail: alberto.zapatera@uchceu.es.
} 
the trainers to interpret the PPT progress in the development of the professional look competence of children's mathematical thinking in the context of pattern generalization.

Keywords: Professional look. Pattern generalization. Descriptors of development. Docent practice. Problem resolution.

\section{Problemática de la investigación}

La competencia docente mirar profesionalmente el pensamiento matemático de los alumnos es una línea de investigación con gran relevancia dentro del desarrollo profesional del profesor de matemáticas (SHERIN; JACOBS; PHILIPP, 2011; BARNHART; VAN ES, 2015). Una manera de conceptualizar la mirada profesional es a partir del estudio de las tres destrezas descritas por Jacobs, Lamb y Philipp (2010): (1) identificar los aspectos relevantes, (2) interpretar la comprensión de los alumnos y (3) tomar decisiones de acción.

Para describir el desarrollo de la mirada profesional, algunas investigaciones han codificado las respuestas de los participantes según niveles de evidencias. En unos casos, la descripción de estos niveles ha sido vaga y poco operativa, con indicadores genéricos y ambiguos (JACOBS; LAMB; PHILIPP, 2010; BARTELL et al., 2013).

En otros casos, la descripción de los niveles ha sido más operativa y se ha basado en la identificación de los elementos matemáticos relevantes en las respuestas de los estudiantes y su uso para interpretar la comprensión (FERNÁNDEZ; VALLS; LIINARES, 2011; SÁNCHEZ-MATAMOROS; FERNÁNDEZ; LLINARES, 2014) o en las etapas de una trayectoria de aprendizaje sobre un contenido concreto (SCHACK et al., 2013; WILSON; MOJICA; CONFREY, 2013). En el Cuadro 1 se resumen algunas de las investigaciones revisadas. 


\begin{tabular}{|c|c|c|c|c|c|}
\hline Referencias & Criterio & Participantes & Soporte & Dominio & Destrezas \\
\hline $\begin{array}{l}\text { Jacobs, Lamb y Philipp } \\
\text { (2010) }\end{array}$ & $\begin{array}{l}\text { Estrategias con } \\
\text { distintos } \\
\text { niveles de } \\
\text { elaboración }\end{array}$ & $\begin{array}{l}\text { EPM y } \\
\text { maestros }\end{array}$ & $\begin{array}{l}\text { Respuestas escritas } \\
\text { y vídeos }\end{array}$ & $\begin{array}{l}\text { Problemas de } \\
\text { estructuras aditiva } \\
\text { y multiplicativa }\end{array}$ & $\begin{array}{l}\text { Identificar } \\
\text { interpretar y } \\
\text { decidir }\end{array}$ \\
\hline $\begin{array}{l}\text { Bartell, Webel, Bowen y } \\
\text { Dyson (2013) }\end{array}$ & $\begin{array}{l}\text { Corrección } \\
\text { respuestas }\end{array}$ & EPM & $\begin{array}{l}\text { Respuestas } \\
\text { escritas, entrevistas } \\
\text { y vídeos }\end{array}$ & $\begin{array}{l}\text { Números } \\
\text { racionales }\end{array}$ & $\begin{array}{l}\text { Identificar e } \\
\text { interpretar }\end{array}$ \\
\hline $\begin{array}{l}\text { Fernández, Valls y } \\
\text { Llinares (2011) }\end{array}$ & $\begin{array}{l}\text { Perfiles de } \\
\text { desarrollo }\end{array}$ & EPM & Respuestas escritas & $\begin{array}{l}\text { Problemas } \\
\text { proporcionales }\end{array}$ & $\begin{array}{l}\text { Identificar, } \\
\text { interpretar y } \\
\text { decidir }\end{array}$ \\
\hline $\begin{array}{l}\text { Sánchez-Matamoros, } \\
\text { Fernández y Llinares } \\
(2014)\end{array}$ & $\begin{array}{l}\text { Niveles teoría } \\
\text { APOS }\end{array}$ & EPP & $\begin{array}{l}\text { Respuestas escritas } \\
\text { y entrevistas }\end{array}$ & Derivada & Interpretar \\
\hline $\begin{array}{l}\text { Schack, Fisher, Thomas, } \\
\text { Eisenhardt, Tassell y } \\
\text { Yoder (2013) }\end{array}$ & $\begin{array}{l}\text { Trayectoria } \\
\text { SEAL }\end{array}$ & EPM & Vídeos & $\begin{array}{l}\text { Introducción a la } \\
\text { aritmética }\end{array}$ & $\begin{array}{l}\text { Identificar, } \\
\text { interpretar y } \\
\text { decidir }\end{array}$ \\
\hline $\begin{array}{l}\text { Wilson, Mojica y Confrey } \\
\text { (2013) }\end{array}$ & $\begin{array}{l}\text { Trayectoria } \\
\text { EPLT }\end{array}$ & Maestros & $\begin{array}{l}\text { Respuestas } \\
\text { escritas, entrevistas } \\
\text { y vídeos }\end{array}$ & $\begin{array}{l}\text { Equivalencia de } \\
\text { fracciones }\end{array}$ & $\begin{array}{l}\text { Describir, } \\
\text { comparar e } \\
\text { inferir }\end{array}$ \\
\hline
\end{tabular}

Cuadro 1 - Ejemplos de investigaciones para caracterizar la mirada profesional Fuente: Elaboración propia

Jacobs, Lamb y Philipp (2010) caracterizaron cada una de las tres destrezas mediante tres categorías, basadas en las evidencias que mostraban maestros y estudiantes para maestro (EPM) al analizar las respuestas de alumnos a problemas aditivos y multiplicativos: evidencias sólidas, algunas evidencias y sin evidencias. Consideraron evidencias de la destreza identificar aspectos matemáticos como la forma en la que los alumnos contaban, utilizaban dibujos o descomponían números; consideraron evidencias de la destreza interpretar si daban detalles de las estrategias y si explicaban cómo esos detalles reflejan el pensamiento de los alumnos; y consideraron evidencias de la destreza decidir si los participantes, a la hora de proponer acciones, tenían en cuenta, de forma explícita, las estrategias utilizadas por los alumnos y, en algunos casos, si se anticipaban a una posible estrategia futura.

Bartell et al. (2013) caracterizaron, de forma conjunta, las destrezas identificación e interpretación en EPM; para ello, analizaron si reconocían evidencias de la comprensión de los alumnos en las estrategias utilizadas en tareas con números racionales. Clasificaron las respuestas de los participantes en cuatro niveles de evidencia: buena evidencia, alguna evidencia, sin evidencia y evidencia errónea. Entendieron como buena evidencia si los participantes utilizaban conceptos adecuados y sus conclusiones eran correctas; como alguna evidencia si utilizaban conceptos adecuados pero llegaban a conclusiones erróneas; sin 
evidencias si se limitaban a hacer comentarios genéricos o simples descripciones; como evidencias erróneas, si demostraban una mala comprensión de los conceptos matemáticos.

Las investigaciones que basaron su estudio en los elementos matemáticos significativos de tópicos concretos se centraron en si los participantes hacían referencias a los elementos matemáticos y si se apoyaban en estos elementos para interpretar la comprensión de los alumnos.

Fernández, Valls y Llinares (2011) analizaron las descripciones que hacían EPM sobre respuestas de alumnos de Primaria a problemas de razonamiento proporcional. Prestaron atención a si mencionaban los elementos matemáticos relevantes en los problemas propuestos y si en sus argumentaciones los relacionaban o no con la corrección de las respuestas. De esta forma, caracterizaron el desarrollo de la competencia docente mirar profesionalmente en cuatro niveles: en el nivel 1 los EPM no discriminan entre problemas proporcionales y no proporcionales; en el nivel 2 discriminan los problemas, pero no justifican las diferencias; en el 3 discriminan los problemas y justifican las diferencias, pero no identifican perfiles en los alumnos; en el nivel 4 discriminan problemas, justifican diferencias e identifican perfiles.

Sánchez-Matamoros, Fernández y Llinares (2014), en el contexto de la derivada, establecieron descriptores del desarrollo de la mirada profesional en función de los elementos matemáticos significativos que identificaban y de los niveles de comprensión exhibidos en términos de la triada (intra, inter y trans) de la Teoría APOE (DUBINSKY, 1991). Propusieron tres perfiles en las destrezas identificación e interpretación, dependiendo si identificaban e interpretaban todos los elementos, algún elemento o ningún elemento y en la destreza decidir establecieron cinco niveles según el tipo de acciones que proponían.

Otras investigaciones han generado descriptores del desarrollo de la mirada profesional, teniendo en cuenta trayectorias de aprendizaje. Para Simon (1995) y Clements y Sarama (2004) las trayectorias de aprendizaje permiten diagnosticar la comprensión de los estudiantes y describir el progreso en términos de crecimiento a través de niveles, proporcionan un feedback para los maestros y mejoran la enseñanza y el aprendizaje de los estudiantes. Estas investigaciones se han apoyado en las características matemáticas propias de cada nivel de la trayectoria y en los saltos cognitivos necesarios para pasar de un nivel a otro.

Schack et al. (2013) se apoyaron en la trayectoria de aprendizaje SEAL (Stages of Early Arithmetic Learning) que propuso Steffe (1992) sobre el conteo y la secuencia numérica. Codificaron la destreza identificar en cuatro categorías según el grado de evidencia: elaborado, destacado, limitado e inexacto; codificaron la destreza interpretar en tres categorías 
según las evidencias de las etapas de la trayectoria: inferior, intermedio y superior; por último, codificaron la destreza decidir en otras tres categorías según la carga cognitiva que requerían las acciones: baja, media y alta.

Wilson, Mojica y Confrey (2013) utilizaron los estadios de la trayectoria de aprendizaje EPLT (Equi Partitioning Learning Trajectory) sobre la equivalencia de fracciones para describir los niveles de las destrezas de la mirada profesional de maestros. Estudiaron si los maestros relacionaban su análisis de las respuestas de alumnos resolviendo problemas de reparto con los distintos estadios de la trayectoria EPLT. Comprobaron que los maestros pueden construir modelos de pensamiento de los alumnos a partir de los procesos de describir, comparar e inferir.

Estas investigaciones sobre la competencia docente mirar profesionalmente el pensamiento matemático de los alumnos se han focalizado en diferentes dominios matemáticos específicos. El dominio elegido para esta investigación ha sido la generalización de patrones, debido a su importancia para introducir el pensamiento algebraico desde los primeros años de escolarización (RADFORD, 2008). A pesar de esta importancia, pocas investigaciones sobre la mirada profesional se han centrado en la generalización de patrones (MOUHAYAR; JURDAK, 2012; MAGIERA; VAN DEN KIOBOOM; MOYER, 2013; ZAPATERA, 2015b; CALLEJO; ZAPATERA, 2017).

Mouhayar y Jurdak (2012) centraron su investigación en la capacidad de profesores de Secundaria para identificar, describir y explicar las acciones de alumnos resolviendo problemas de generalización de patrones a partir de las estrategias utilizadas. Magiera, van den Kieboom y Moyer (2013) utilizaron algunos problemas de generalización de patrones para estudiar cómo estudiantes para profesor reconocían e interpretaban las características del pensamiento algebraico de los alumnos. Zapatera (2015b) y Callejo y Zapatera (2017) utilizaron problemas de generalización de patrones para establecer cinco perfiles de desarrollo de la mirada profesional en EPM.

Esta investigación complementa las investigaciones sobre la mirada profesional porque, por una parte, estudia la competencia en el contexto de la generalización de patrones, que ha sido poco estudiado a pesar de su importancia para la introducción del pensamiento algebraico temprano, y, por otra parte, enfatiza las dimensiones matemática, cognitiva y didáctica, al analizar cómo futuros maestros usan los elementos matemáticos relevantes identificados en el proceso de generalización para interpretar la comprensión de los alumnos y proponer acciones para mejorar su aprendizaje.

En este contexto, el objetivo de esta investigación es generar descriptores del 
desarrollo de la competencia mirar profesionalmente el pensamiento matemático de los alumnos de Primaria, en futuros maestros, en el marco de la generalización de patrones.

\section{Marco teórico}

\subsection{La mirada profesional como competencia docente}

Un objetivo de la investigación en didáctica de las matemáticas es la formación y desarrollo del profesor de matemáticas, y, en particular, el estudio del conocimiento y de las destrezas que necesita para desarrollar su profesión. De este interés han surgido modelos y corrientes de investigación como la competencia docente mirada profesional (profesional noticing) (MASON, 2002; SHERIN; JACOBS; PHILIPP, 2011).

La mirada profesional, o profesional noticing, es una expresión que se usa para indicar el acto de observar o reconocer sucesos relevantes de una situación y actuar en función de ellos. En este sentido, Mason (2002) considera que la mirada profesional es la capacidad de los maestros para interpretar situaciones de enseñanza-aprendizaje y contempla dos aspectos de la competencia: darse cuenta de (account of), que se centra en observar las situaciones, evitando interpretaciones, juicios o evaluaciones y darse cuenta para (account for), que se centra en interpretar y explicar lo que se observa para adoptar decisiones adecuadas.

Siguiendo esta línea, Van Es (2011) propone un marco sobre la mirada profesional, diferenciando dos dimensiones: qué observan los maestros y cómo lo interpretan. La primera dimensión se refiere a las evidencias identificadas en las estrategias pedagógicas, en el comportamiento y en el pensamiento matemático de los alumnos, y en la segunda dimensión se incluyen las impresiones, valoraciones e interpretaciones que realizan los maestros acerca del pensamiento de los alumnos.

Jacobs, Lamb y Phillip (2010) centran la mirada profesional en el pensamiento matemático de los estudiantes, y consideran que la mirada profesional implica identificar los hechos relevantes e interpretarlos para dotarlos de significado y poder decidir acciones para mejorar el proceso de enseñanza y aprendizaje. De esta manera, conceptualizan esta competencia como el conjunto de tres destrezas: (1) identificar las estrategias que utilizan los estudiantes, (2) interpretar la comprensión de los estudiantes a partir de las estrategias identificadas y (3) decidir cómo responder con base en la comprensión de los estudiantes.

La primera destreza implica la identificación de los aspectos matemáticos significativos en las estrategias que usan los estudiantes al resolver una determinada tarea 
matemática; a veces estas estrategias son complejas y difíciles de identificar, pero sus detalles son importantes porque proporcionan una ventana para mirar el pensamiento matemático de los alumnos (CARPENTER; FRANKE; LEVI, 2003).

En la segunda destreza el maestro, para interpretar la comprensión matemática de los estudiantes, debe conectar los elementos matemáticos significativos y las estrategias identificadas en las respuestas de los alumnos con aspectos cognitivos. Algunas investigaciones consideran que tan importante como lo que se observa es la forma en la que se interpreta lo observado (SHERIN; VAN ES, 2005; ZAPATERA, 2015a).

En la tercera destreza el maestro utiliza la interpretación de la comprensión de los alumnos que ha realizado a partir de los elementos matemáticos y las estrategias identificadas en sus respuestas, para decidir las acciones y mejorar el proceso de enseñanza-aprendizaje. Van Es y Sherin (2002) subrayan este aspecto práctico de la competencia mirada profesional al señalar que lo que se mira no puede ser separado del objetivo que se pretende.

Jacobs, Lamb y Philipp (2010) sostienen que las tres destrezas están interrelacionadas y anidadas, ya que, para tomar decisiones adecuadas, es necesario interpretar la comprensión de los estudiantes y esta interpretación debe apoyarse en la identificación de los elementos matemáticos significativos.

\subsection{Generalización de patrones}

Numerosos investigadores en Educación Matemática han definido la generalización. Por ejemplo, Pólya (1954, p. 12) subraya la acción de extender e indica que generalizar es "pasar de un objeto a una clase que contiene el objeto"; Harel y Tall (1991, p. 38) consideran que generalizar es "aplicar un argumento dado en un contexto más amplio"; y Dreyfus (1991, p. 35) señala que generalizar es "derivar o inducir desde lo particular, identificando lo que es común y extendiendo dominios de validez para incluir un conjunto mayor de casos”.

En el caso específico de la generalización de patrones, es decir, en tareas en las que se trata de identificar un patrón en una sucesión de la que se expresan gráficamente los primeros términos, Radford (2008) considera tres aspectos: (1) darse cuenta de una propiedad común de los términos de la secuencia, (2) extender la propiedad a todos los términos de la secuencia y (3) encontrar una regla para calcular cualquier término de la secuencia.

Investigadores que se han centrado en cómo resuelven los alumnos de Primaria tareas de generalización de patrones (CARRAHER; MARTÍNEZ; SCHLIEMANN, 2008; RADFORD, 2008; RIVERA, 2010; WARREN, 2005), han destacado el papel relevante de 
tres elementos matemáticos: estructuras numérica y espacial, relación funcional y relación funcional inversa.

Las estructuras numérica y espacial emergen, respectivamente, del número de elementos de cada término o figura de la sucesión y de la distribución de esos elementos (RADFORD, 2014); la relación funcional asocia el término de la sucesión con el número de elementos que contiene (RADFORD, 2008; RIVERA, 2010; WARREN, 2005); y la función inversa permite hallar el término de la sucesión a partir del número de elementos (WARREN, 2005).

Del uso de estos elementos matemáticos en la resolución de problemas de generalización de patrones se infieren los aspectos cognitivos, necesarios para que los alumnos comprendan el proceso de generalización: coordinación entre estructuras espacial y numérica, pensamiento funcional y reversibilidad (ZAPATERA; CALLEJO, 2018). El alumno coordina ambas estructuras cuando identifica una regularidad que las relaciona; muestra su pensamiento funcional cuando relaciona de forma numérica, verbal o algebraica, cualquier término de la sucesión con el número de elementos que la forma; y domina la reversibilidad cuando es capaz de reconocer la posición en la secuencia de un determinado término a partir del número de elementos de dicho término.

A partir de los elementos matemáticos y los aspectos cognitivos se han identificado tres estadios de la comprensión de la generalización de patrones en los alumnos de Primaria: en el estadio 1, el alumno, aunque puede continuar la sucesión para términos cercanos, no coordina las estructuras numérica y espacial, lo que le impide calcular elementos de términos lejanos; para pasar al estadio 2, el alumno debe coordinar las estructuras espacial y numérica, lo que le permitirá reconocer una relación funcional y expresarla en una regla general para hallar el número de elementos de cualquier término de la sucesión, pero no invierte el proceso; para pasar al estadio 3, el alumno debe identificar la relación inversa, lo que le permitirá invertir la relación en casos específicos (CALLEJO; ZAPATERA, 2017) (Figura 1).

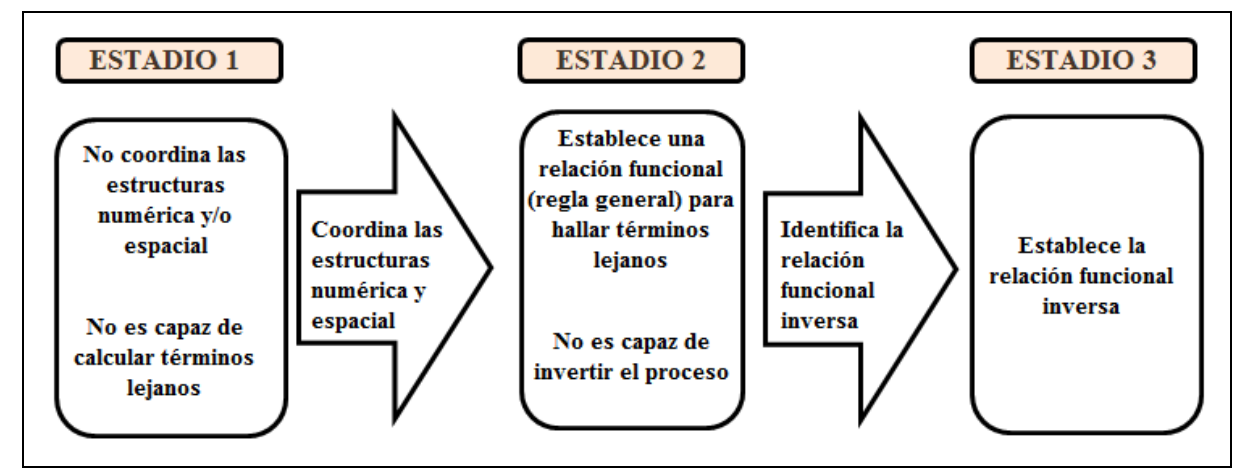

Figura 1 - Estadios de comprensión Fuente: Zapatera (2018) 
Estos estadios establecen una progresión en el desarrollo del proceso de generalización que se describe mediante los saltos cognitivos necesarios para pasar de un estadio a otro. Esta progresión puede ayudar a detectar las dificultades con las que se encuentran los estudiantes y, desde el punto de vista didáctico, puede servir como punto de apoyo para mejorar el proceso de enseñanza-aprendizaje de los alumnos.

Para generar los descriptores del desarrollo de la competencia mirar profesionalmente el pensamiento matemático de los alumnos de Primaria, en futuros maestros, en el marco de la generalización de patrones, se plantean tres preguntas de investigación encadenadas: (1) ¿identifican los EPM los elementos matemáticos en las respuestas de los alumnos de Primaria?, (2) ¿utilizan los EPM los elementos matemáticos identificados para interpretar los aspectos cognitivos de la comprensión del pensamiento matemático de los alumnos? y (3) ¿los aspectos cognitivos ayudan a los EPM a proponer acciones didácticas para mejorar o ampliar el proceso enseñanza-aprendizaje de los alumnos de Primaria?

\section{Método}

\subsection{Participantes y contexto}

En esta investigación participaron veinte estudiantes para maestro (EPM) que cursaban la asignatura Aprendizaje y didáctica de las matemáticas del tercer curso del Grado en Maestro en Educación Primaria, cuyo programa de formación abarca cuatro cursos.

La asignatura incluía la realización de un experimento de enseñanza de siete sesiones cuyo objetivo era desarrollar la competencia mirar profesionalmente el pensamiento matemático de los alumnos en el contexto de la generalización de patrones. En el experimento resolvieron problemas de generalización de patrones, estudiando diferentes estrategias de resolución (estrategias aditivas, funcionales y de proporcionalidad) y se les proporcionó información sobre las destrezas de la mirada profesional en el contexto de la generalización de patrones.

Esta información se centró, principalmente, en que los EPM reconocieran los elementos matemáticos significativos (estructuras espacial y numérica, relación funcional y relación funcional inversa) vinculados a los tres aspectos cognitivos (coordinación de estructuras, pensamiento funcional y reversibilidad del pensamiento funcional) que permiten establecer los tres estadios de comprensión de los alumnos de Primaria. 


\subsection{Instrumentos de recogida de datos}

Los EPM analizaron las respuestas de tres alumnos de Educación Primaria a un problema de generalización de patrones (Figura 2) adaptado de Carraher, Martínez y Schliemann (2008). El problema presenta una situación cuyo enunciado proporciona los primeros términos de una sucesión formada por mesas y sillas en la que se pide a los alumnos (1) continuar la sucesión y calcular el número de sillas para un número pequeño de mesas (generalización cercana), (2) calcular el número de sillas para un número grande de mesas (generalización lejana), (3) explicar la regla general que relacione las dos variables, es decir, sillas y mesas y (4) calcular el número de mesas para un determinado número de sillas (proceso inverso)

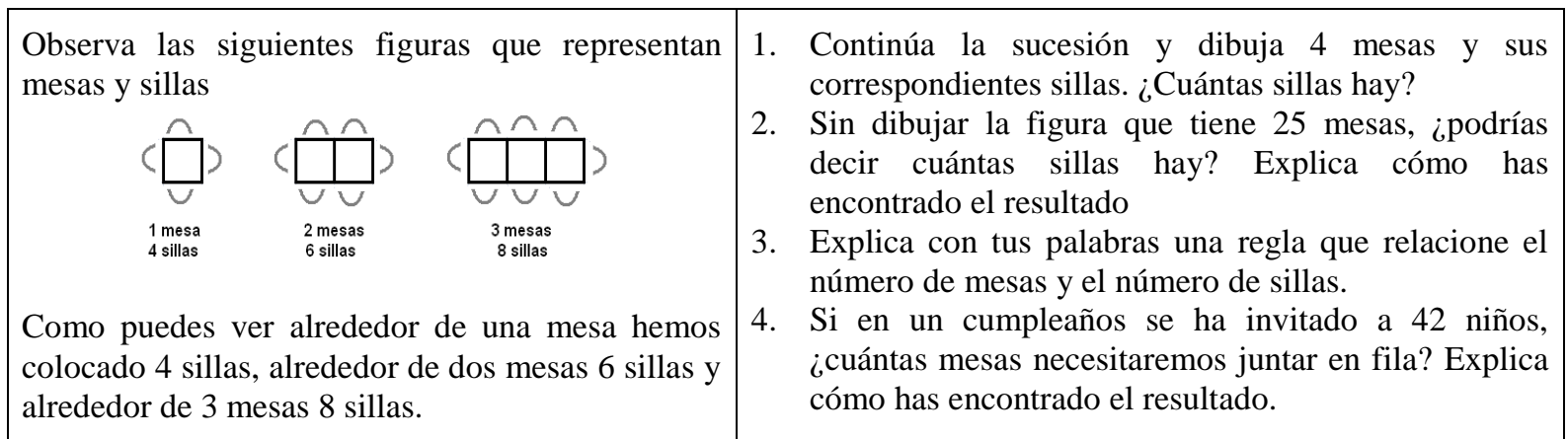

Figura 2 - Problema de generalización de patrones Fuente: Elaboración propia

En la Figura 3 se recogen las respuestas de tres alumnos de Educación Primaria al problema de generalización de patrones. Estas respuestas fueron seleccionadas entre las respuestas de alumnos de $3^{\circ}, 4^{\circ}, 5^{\circ}$ y $6^{\circ}$ de Educación Primaria que participaron en una investigación previa sobre la generalización de patrones (CALLEJO; ZAPATERA, 2017).

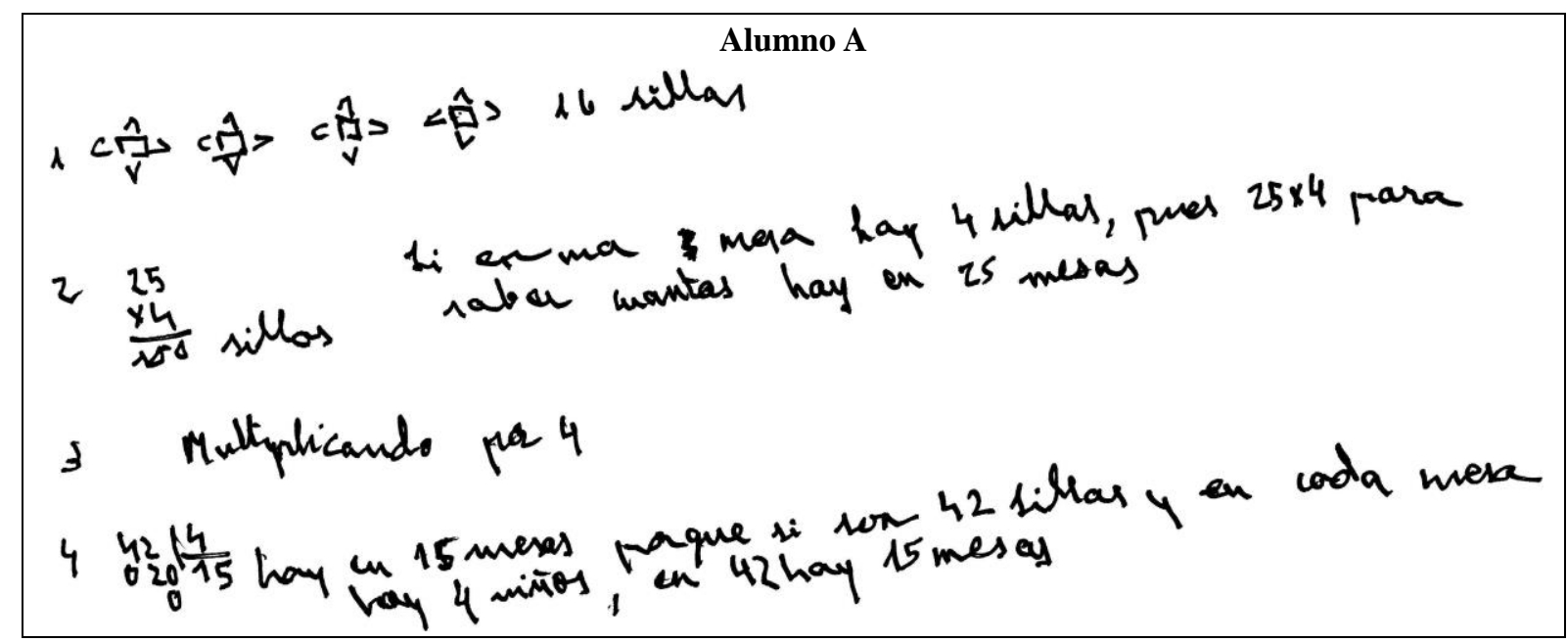



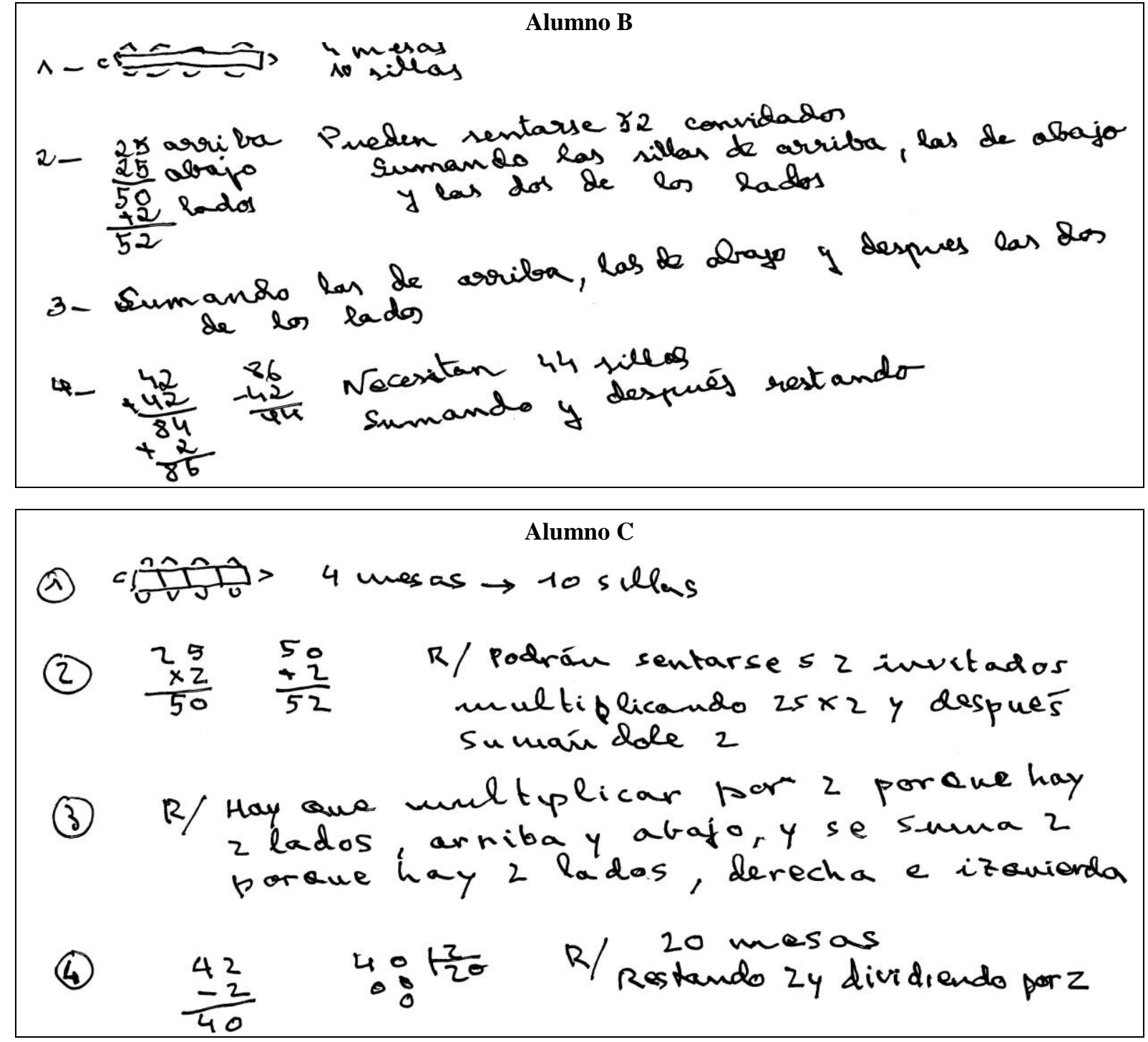

Figura 3 - Respuestas de alumnos de Primaria al problema de generalización de patrones Fuente: Elaboración propia

El alumno A pertenece al primer estadio, ya que no es capaz de coordinar las estructuras espacial y numérica, lo que provoca que defina una relación funcional errónea que le impide resolver correctamente el resto de apartados; el alumno B pertenece al estadio 2, ya que coordina las estructuras espacial y numérica, lo que le permite establecer una relación funcional de forma verbal para hallar los elementos de términos lejanos, pero no es capaz de invertir el proceso, es decir, no calcula el número de mesas dado el número de sillas; el alumno C está en el último estadio de comprensión, puesto que coordina las estructuras, establece una relación funcional y, además, es capaz de invertir la relación funcional.

Para analizar las respuestas de los tres alumnos de Primaria, los EPM contestaron un cuestionario con tres tareas relacionadas con las tres destrezas de Jacobs, Lamb y Philipp (2010) (Cuadro 2). 
1. Identifica en las respuestas de cada alumno los aspectos del proceso de generalización de patrones que consideres más interesantes

2. Interpreta la comprensión del proceso de generalización de patrones de cada alumno a partir de los aspectos identificados

3. Propón acciones para mejorar o ampliar la comprensión del proceso de generalización de patrones de cada alumno a partir de las interpretaciones realizadas

Cuadro 2 - Tareas del cuestionario 2

Fuente: Elaboración propia

El objetivo de la primera tarea era ver en qué medida los EPM identifican los elementos matemáticos significativos usados por los alumnos de Primaria; con la segunda tarea se pretendía determinar en qué medida los EPM interpretan las características de la comprensión del proceso de generalización de cada uno de los alumnos de Primaria a partir de los aspectos cognitivos y estadios de comprensión vinculados a los elementos matemáticos identificados; con la tercera tarea se buscaba determinar qué decisiones de acción toman los EPM en función de las interpretaciones realizadas para mejorar o ampliar el proceso de enseñanza-aprendizaje de los alumnos.

Con estas tres cuestiones, relacionadas directamente con las tres destrezas que conceptualizan la mirada profesional, se pretendía abarcar, también, tres dimensiones de la enseñanza de las matemáticas: la dimensión epistemológica o matemática, asociada a las características del saber matemático; la dimensión cognitiva, asociada a las características cognitivas del alumno; y la dimensión didáctica, asociada a las características del sistema de enseñanza (ARTIGUE, 1995).

La primera cuestión abarca la dimensión matemática asociada a las características del saber matemático porque, para identificar los elementos matemáticos significativos, el maestro debe tener un suficiente conocimiento matemático de la generalización de patrones; la segunda cuestión abarca la dimensión cognitiva asociada a las caracteríaticas cognitivas de los alumnos porque, para interpretar la comprensión de los alumnos, el maestro debe conocer los aspectos cognitivos y estadios de comprensión de la generalización de patrones; y la tercera cuestión abarca la dimensión didáctica asociada a las características del sistema de eneseñanza porque, para decidir acciones adecuadas, el maestro debe conocer las tareas matemáticas que ayuden al avance conceptual de los alumnos en el tópico de la generalización de patrones (SÁNCHEZ-MATAMOROS et al., 2013; ZAPATERA; CALLEJO, 2013; CALLEJO; ZAPATERA, 2017).

\subsection{Análisis de datos}

El análisis de datos se realizó desde la perspectiva de la Teoría Fundamentada, 
utilizando el método de la comparación constante de Strauss y Corbin (1994). Para asegurar la validez y fiabilidad del análisis, tres investigadores analizaron una pequeña muestra, discutieron las codificaciones y acordaron las relaciones entre las evidencias y las codificaciones. Después, por un proceso inductivo de saturación y refinamiento de los códigos, se identificaron diferentes grupos de EPM según su nivel de desarrollo de la competencia docente y a partir de estos grupos se generaron descriptores del desarrollo de la mirada profesional.

Los datos se analizaron desde las tres dimensiones: matemática, cognitiva y didáctica. Desde la dimensión matemática se tuvo en cuenta si los EPM identificaban, de forma explícita o implícita, los elementos matemáticos relevantes de la generalización de patrones (estructuras espacial y numérica, relación funcional y relación funcional inversa) cuando describían las respuestas de los alumnos. Por ejemplo, el EPM-3, en la primera tarea profesional, identificó las estructuras espacial y numérica al observar que:

Este alumno no respeta la distribución espacial porque no dibuja igual que el ejemplo y además dibuja más sillas de las que le pide el ejercicio (EPM-3, 2017).

Desde la dimensión cognitiva se tuvo en cuenta si los EPM utilizaban los tres aspectos cognitivos de la comprensión de la generalización (coordinación entre las estructuras espacial y numérica, pensamiento funcional y reversibilidad del pensamiento funcional) y los estadios de comprensión asociados a ellos para interpretar la comprensión de los alumnos. Por ejemplo, el EPM-19 para interpretar la comprensión del alumno del estadio 1 utilizó la coordinación de estructuras y el pensamiento funcional del alumno al afirmar que:

Este alumno no respeta la estructura espacial ni la numérica ya que dibuja las mesas separadas y debido a ello no coordina la espacial con la numérica, lo que le impide relacionar correctamente las mesas con las sillas (EPM-19, 2017).

Desde la dimensión didáctica se consideró si las acciones que proponían los EPM hacían o no referencia a los elementos matemáticos y a los aspectos cognitivos relevantes para superar las dificultades de los alumnos A y B o para ampliar los conocimientos del alumno C. Por ejemplo, el EPM-11 tuvo en cuenta la necesidad de coordinar las estructuras espacial y numérica al interpretar el pensamiento del alumno del estadio 1 cuando expresó que:

Como tiene dificultades en la representación y recuento de mesas y sillas, propondría hacer ejercicios manipulativos de seriaciones en los que tenga que mantener el esquema espacial de los primeros términos de la secuencia y con este tipo de ejercicios el alumno mejoraría la coordinación entre la distribución numérica y los elementos de cada figura (EPM-11, 2017).

El análisis realizado permitió identificar y caracterizar cuatro niveles de desarrollo de la mirada profesional en los EPM en el contexto de la generalización de patrones (Cuadro 3). 


\begin{tabular}{|l|l|}
\hline Nivel & Caracterización \\
\hline Nivel 0 & EPM que no identifican elementos matemáticos \\
\hline Nivel 1 & $\begin{array}{l}\text { EPM que identifican elementos matemáticos, pero no los usan para interpretar la comprensión } \\
\text { de los alumnos }\end{array}$ \\
\hline Nivel 2 & $\begin{array}{l}\text { EPM que identifican elementos matemáticos y los usan para interpretar la comprensión de los } \\
\text { alumnos, pero no proponen acciones adecuadas para mejorar o ampliar el proceso }\end{array}$ \\
\hline Nivel 3 & $\begin{array}{l}\text { EPM que identifican elementos matemáticos, los usan para interpretar la comprensión de los } \\
\text { alumnos y proponen acciones adecuadas para mejorar o ampliar el proceso }\end{array}$ \\
\hline
\end{tabular}

Cuadro 3 - Caracterización de los niveles

Fuente: Elaboración propia

\section{Resultados}

La asignación de niveles de desarrollo de la mirada profesional en los EPM se realizó, inicialmente, de forma individualizada para cada alumno y, después, se ponderaron los resultados de cada EPM para asignarle un nivel general, de forma que el nivel asignado fue el obtenido por cada EPM en al menos dos alumnos (Figura 4).

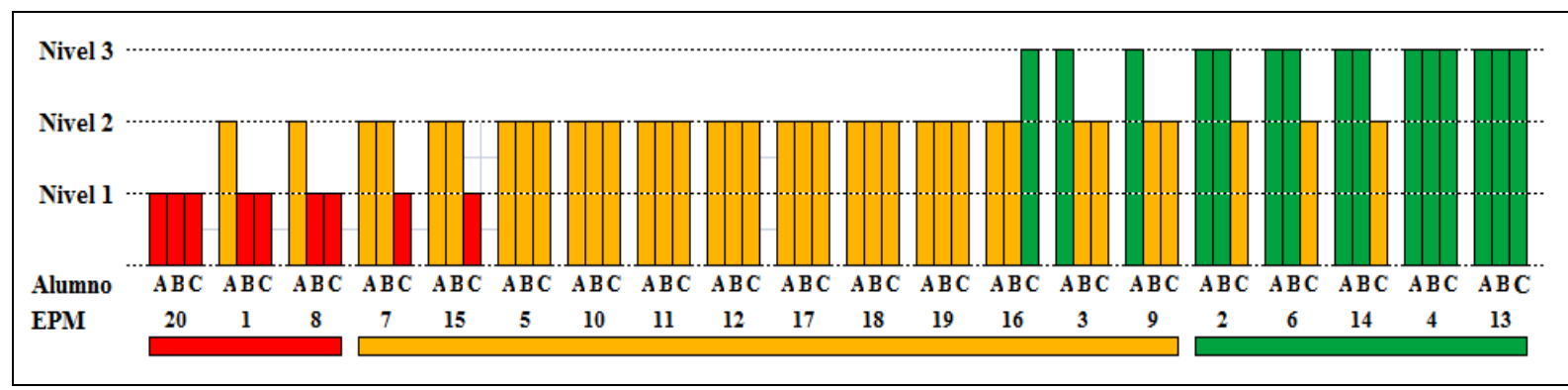

Figura 4 - Niveles de los EPM

Fuente: Elaboración propia

Al ponderar los niveles obtenidos por cada EPM al analizar las respuestas de los tres alumnos de Primaria, se observa que ningún EPM está en el nivel 0, que tres EPM están en el nivel 1, doce EPM en el nivel 2 y cinco EPM en el nivel 3:

- $\quad$ El nivel 0 no ha sido asignado a ningún EPM porque todos han identificado, implícita o explícitamente, algún elemento matemático relevante en los tres alumnos.

- Los tres EPM del nivel 1 han identificado algún elemento matemático en los tres alumnos, pero uno no los ha usado para interpretar la comprensión de ninguno de ellos y los otros dos solo los han usado para interpretar la comprensión del alumno A, del estadio 1.

- De los doce EPM del nivel 2, dos han usado los elementos para interpretar la comprensión de los alumnos A y B y los otros diez EPM los han usado para interpretar a los tres alumnos; tres EPM, además de identificar elementos e interpretar la 
comprensión de los tres alumnos, han propuesto acciones concretas para algún alumno.

- De los cinco EPM del nivel 3, tres no han propuesto acciones concretas para el alumno $\mathrm{C}$, mientras que los otros dos EPM sí fueron capaces de proponer acciones concretas para los tres alumnos.

A los EPM les ha resultado fácil identificar los elementos matemáticos relevantes, pero no les ha resultado tan fácil usarlos para interpretar la comprensión de los alumnos, y les ha resultado aún más difícil proponer acciones para mejorar el rendimiento: todos los EPM han identificado los elementos matemáticos de los tres alumnos; quince EPM han utilizado los elementos matemáticos para interpretar la comprensión de los tres alumnos; y solo dos EPM han propuesto acciones adecuadas para mejorar o ampliar el conocimiento de los tres alumnos.

Aunque todos los EPM han identificado los elementos matemáticos en las respuestas de los tres alumnos, el alumno mejor analizado ha sido el alumno A, del primer estadio, y el peor, el alumno C, del tercer estadio: diecinueve EPM han interpretado la comprensión del alumno A, diecisiete la del alumno B y quince la del alumno C; y siete EPM han propuesto acciones adecuadas para el alumno A, cinco para el B y dos para el C.

A continuación se comentan las características y los resultados obtenidos por los EPM de cada nivel y se muestran, a modo de ejemplo, las respuestas de algunos de ellos.

\subsection{Nivel 1 - EPM que identifican elementos matemáticos, pero no los usan para interpretar la comprensión de los alumnos}

Los tres EPM de este nivel han identificado, implícita o explícitamente, el elemento matemático de las estructuras espacial y numérica en el alumno A, al reconocer que no ha dibujado correctamente las mesas; han identificado que el alumno B coordina las estructuras y establece la relación funcional, pero no invierte el proceso; en el alumno $\mathrm{C}$ han identificado que coordina las estructuras, establece la relación funcional e invierte el proceso.

Sin embargo, no han utilizado la identificación de los elementos para interpretar la comprensión de los alumnos ni para proponer acciones adecuadas para mejorar el proceso: un EPM no los ha utilizado en ninguno de los tres alumnos y los otros dos EPM solo los ha utilizado para interpretar la comprensión del alumno A.

El EPM-20 se encuentra en el nivel 1 en el anáisis del alumno A: ha reconocido implícitamente la falta de coordinación entre estructuras, pero no ha sido capaz de interpretar 
la comprensión del alumno y ha propuesto una acción de tipo actitudinal, sin relación con la generalización de patrones (Cuadro 4).

\begin{tabular}{|c|c|c|c|}
\hline Alumno & EPM & Destreza & Análisis \\
\hline \multirow[t]{3}{*}{ A } & \multirow[t]{3}{*}{20} & Identificación & $\begin{array}{l}\text { Ha dibujado mal las mesas porque las ha separado y no debería } \\
\text { haberlo hecho. }\end{array}$ \\
\hline & & Interpretación & No ha sabido hacer bien el problema. \\
\hline & & Decisión & $\begin{array}{l}\text { No ha sabido hacer ningún apartado bien. Le ayudaría para que no se } \\
\text { desanimara y siguiera intentándolo. }\end{array}$ \\
\hline \multirow[t]{3}{*}{ B } & \multirow[t]{3}{*}{1} & Identificación & $\begin{array}{l}\text { Ha dibujado bien las mesas y las sillas y ha expresado correctamente la } \\
\text { regla, pero no ha sabido hacer lo contrario en la pregunta } 4 .\end{array}$ \\
\hline & & Interpretación & $\begin{array}{l}\text { Ha hecho todo el problema bien, menos la pregunta 4; no está mal para } \\
\text { su edad. }\end{array}$ \\
\hline & & Decisión & $\begin{array}{l}\text { Este alumno no entiende el enunciado, lo que puede ser debido a que no } \\
\text { lo ha leído bien, le recomendaría leer más y que no se desanime. }\end{array}$ \\
\hline \multirow[t]{3}{*}{$\mathrm{C}$} & \multirow[t]{3}{*}{8} & Identificación & $\begin{array}{l}\text { Domina todos los elementos de la generalización: estructuras, regla y } \\
\text { proceso inverso. }\end{array}$ \\
\hline & & Interpretación & Ha resuelto bien todas las cuestiones, ha superado el tema. \\
\hline & & Decisión & $\begin{array}{l}\text { Para que este niño avance y amplie su conocimiento le propondría } \\
\text { ejercicios más difíciles ya que estos ya los tiene bien asimilados. }\end{array}$ \\
\hline
\end{tabular}

Cuadro 4 - Ejemplos de análisis de EPM del nivel 1 Fuente: Elaboración propia

El EPM-1, aunque ha identificado implícitamente los tres elementos en el alumno B, no los ha usado ni para interpretar su comprensión ni para proponer acciones adecuadas para mejorar su rendimiento. En la interpretación se limita a decir que no está mal para su edad y en la toma de decisiones le recomienda leer más y que no se desanime (Cuadro 4).

El EPM-8 considera que el alumno C domina el proceso de generalización de patrones y enumera los elementos matemáticos significativos, pero no los usa para interpretar su comprensión, limitándose a decir que ha resuelto bien todas las cuestiones y, aunque reconoce la necesidad de ampliar sus conocimientos, no propone ninguna acción concreta para ello (Cuadro 4).

\subsection{Nivel 2 - EPM que identifican elementos matemáticos y los usan para interpretar la comprensión de los alumnos, pero no proponen acciones adecuadas para mejorar o ampliar el proceso}

La mayoría de los EPM participantes se encuentran en el nivel 2 en los tres alumnos, es decir, han identificado los elementos matemáticos y los han utilizado para interpretar la comprensión de los tres alumnos, pero no han sido capaces de proponer acciones adecuadas para mejorar el rendimiento y la progresión de los alumnos: solo dos de los doce EPM de este nivel han propuesto acciones adecuadas para superar la dificultad del alumno A y un tercero 
ha propuesto acciones para ampliar el conocimiento del alumno $\mathrm{C}$.

El EPM-15 ha identificado el elemento de las estructuras espacial y numérica y lo ha usado para interpretar la comprensión del alumno A, al considerar que la falta de coordinación le impide progresar en la generalización de patrones, pero propone una acción poco específica, que prestara más atención y que mejorara su comprensión lectora, para superar la dificultad (Cuadro 5).

\begin{tabular}{|c|c|c|c|}
\hline Alumno & EPM & Destreza & Análisis \\
\hline \multirow[t]{3}{*}{ A } & \multirow[t]{3}{*}{15} & Identificación & $\begin{array}{l}\text { Este alumno no sigue bien la secuencia de figuras, es decir, no respeta } \\
\text { la distribución espacial porque separa las mesas. }\end{array}$ \\
\hline & & Interpretación & $\begin{array}{l}\text { No puede seguir la secuencia porque no coordina las estructuras } \\
\text { espacial y numérica, lo que le impide progresar en el tema. }\end{array}$ \\
\hline & & Decisión & $\begin{array}{l}\text { A este alumno le diría que prestara más atención y que mejorara su } \\
\text { comprensión lectora. }\end{array}$ \\
\hline \multirow[t]{3}{*}{ B } & \multirow[t]{3}{*}{16} & Identificación & $\begin{array}{l}\text { Ha resuelto correctamente el problema, menos el último apartado. Ha } \\
\text { hecho bien las estructuras y la regla la ha explicado muy bien. }\end{array}$ \\
\hline & & Interpretación & $\begin{array}{l}\text { Está en el estadio } 2 \text { porque coordina las estructuras y sabe hallar } \\
\text { relación pero no sabe hacer el caso contrario. }\end{array}$ \\
\hline & & Decisión & $\begin{array}{l}\text { Como el fallo de este alumno es que no entiende que en el último } \\
\text { apartado le piden lo contrario le pondría ejercicios en los que se } \\
\text { trabajar el proceso inverso. }\end{array}$ \\
\hline \multirow[t]{3}{*}{$\mathrm{C}$} & \multirow[t]{3}{*}{10} & Identificación & $\begin{array}{l}\text { Este alumno ha coordinado correctamente las estructuras, ha hallado la } \\
\text { relación existente entre el número de mesas y de sillas y ha sabido } \\
\text { realizar el proceso inverso. }\end{array}$ \\
\hline & & Interpretación & $\begin{array}{l}\text { Este alumno está en el estadio } 3 \text { porque controla todos los apartados de } \\
\text { la generalización de patrones. }\end{array}$ \\
\hline & & Decisión & $\begin{array}{l}\text { Este alumno lo entiende todo y responde bien a todos los apartados por } \\
\text { lo que le felicitaría y le animaría a que siguiera de esa manera. }\end{array}$ \\
\hline
\end{tabular}

Fuente: Elaboración propia

El EPM-16 ha identificado los elementos y los ha utilizado para clasificar al alumno B en el estadio 2, explicando las características de este estadio, pero no ha propuesto ninguna acción concreta para superar la dificultad del alumno; a pesar de ser consciente del fallo del alumno que no entiende el último apartado, se limita a proponer ejercicios para trabajar el proceso inverso (Cuadro 5).

El EPM-10 ha identificado los elementos matemáticos y los ha interpretado para asignar al alumno $\mathrm{C}$ el estadio 3, sin embargo no ha propuesto ninguna acción para ampliar su conocimiento y se ha limitado a decir que le felicitaría y le animaría a que siguiera de esa manera (Cuadro 5).

\subsection{Nivel 3 - EPM que identifican elementos matemáticos, los usan para interpretar la comprensión de los alumnos y proponen acciones adecuadas para mejorar o ampliar el proceso}


Solo cinco EPM han alcanzado el nivel 3; la mayor dificultad de los EPM se localiza al proponer acciones concretas para mejorar el rendimiento de los alumnos, es decir, superar la falta de coordinación de estructuras en el alumno A, la dificultad para invertir el proceso del alumno B y, especialmente, para ampliar los concimientos del alumno C.

El EPM-2 reconoce que el alumno A se encuentra en el estadio 1 de comprensión porque no domina la estructura espacial, lo que le impide resolver bien las demás cuestiones y propone una acción concreta para que, manipulando materiales, pueda mejorar su comprensión de la generalización y ver mejor la coordinación entre lo espacial y lo numérico (Cuadro 6).

\begin{tabular}{|c|c|c|c|}
\hline Alumno & EPM & Destreza & Análisis \\
\hline \multirow[t]{3}{*}{ A } & \multirow[t]{3}{*}{2} & Identificación & $\begin{array}{l}\text { Dibuja la sucesión de patrones de manera incorrecta porque dibuja las } \\
\text { mesas separadas por lo que tiene sillas de más. }\end{array}$ \\
\hline & & Interpretación & $\begin{array}{l}\text { Presenta un grado de comprensión bajo, situándolo en el estadio } 1 \text { ya } \\
\text { que al hacer la estructura espacial mal implica que el resto de } \\
\text { ejercicios lo resuelva mal. }\end{array}$ \\
\hline & & Decisión & $\begin{array}{l}\text { Para que mejore su comprensión y visualice mejor el problema } \\
\text { utilizaría materiales manipulativos o cercanos al niño, como son las } \\
\text { mesas y sillas de clase [...] De esta manera vería mejor la coordinación } \\
\text { entre lo espacial y lo numérico. }\end{array}$ \\
\hline \multirow[t]{3}{*}{ B } & \multirow[t]{3}{*}{6} & Identificación & $\begin{array}{l}\text { Casi todo bien: dibuja bien las figuras, escribe bien la regla, sumando } \\
\text { las sillas de todos los lados, pero después se lía al hacer el problema } \\
\text { contrario. }\end{array}$ \\
\hline & & Interpretación & $\begin{array}{l}\text { Está en el estadio } 2 \text { porque coordina estructuras, establece relación } \\
\text { funcional, pero no domina el proceso inverso. }\end{array}$ \\
\hline & & Decisión & $\begin{array}{l}\text { Para que entendiera que le piden lo contrario haría ejercicios en los } \\
\text { que se pida una cosa y su inversa. Por ejemplo haría ejercicios de } \\
\text { sumas y a continuación de resta, para que se diera cuenta que es lo } \\
\text { contrario, es decir, primero le plantearía la suma } 8+3 \text { y luego la resta } \\
11-3 \text {. También le haría actividades de multiplicar y luego de dividir, por } \\
\text { ejemplo primero } 4 x 3 \text { y luego 12:3. }\end{array}$ \\
\hline \multirow[t]{3}{*}{$\mathrm{C}$} & \multirow[t]{3}{*}{13} & Identificación & $\begin{array}{l}\text { El alumno C dibuja las mesas y sillas siguiendo la secuencia, para } \\
\text { hallar las sillas multiplica por } 2 \text { las mesas y después suma las } 2 \text { de los } \\
\text { extremos y para hallar las mesas hace lo contrario, primero resta } 2 \text { y } \\
\text { después suma } 2 \text {. }\end{array}$ \\
\hline & & Interpretación & $\begin{array}{l}\text { El alumno establece la estructura espacial y numérica, llega a la } \\
\text { relación funcional e invierte las operaciones, es decir, identifica la } \\
\text { relación funcional, por lo que se encuentra en el estadio } 3 \text {. }\end{array}$ \\
\hline & & Decisión & $\begin{array}{l}\text { Para el alumno } C \text {, que hace bien todo el problema, propondría que } \\
\text { representara las mesas y las sillas con letras, por ejemplo m y s, para } \\
\text { así iniciarle en el lenguaje algebraico. La regla quedaría como } s=2 m \\
+2 \text {. }\end{array}$ \\
\hline
\end{tabular}

Cuadro 6 - Ejemplos de análisis de EPM del nivel 2

Fuente: Elaboración propia

El EPM-6 ha identificado los tres elementos matemáticos y sus respectivos aspectos cognitivos en el alumno B y, además, ha sido capaz de proponer una acción concreta adecuada para mejorar su rendimiento. Como la dificultad del alumno B radica en la reversibilidad del proceso, propone trabajar numéricamente operaciones contrarias: suma-resta y multiplicación- 
división (Cuadro 6).

El EPM-13, para analizar al alumno C, describe sus respuestas identificando las estrategias usadas, utiliza los elementos matemáticos para interpretar su comprensión y asignarle al estadio 3. Para ampliar su conocimiento propone iniciarle en el lenguaje algebraico (Cuadro 5).

\section{Consideraciones finales}

El objetivo de esta investigación era generar descriptores del desarrollo de la competencia mirar profesionalmente el pensamiento matemático de los alumnos en estudiantes para maestro, analizando respuestas de alumnos de Primaria a problemas de generalización de patrones.

Los descriptores se han obtenido a partir del análisis sucesivo de las tres destrezas de Jacobs, Lamb y Philipp (2010): primero se observó si los EPM identificaban los elementos matemáticos en las respuestas de los alumnos de Primaria; después, si utilizaban los elementos identificados para interpretar la comprensión de los alumnos, y, por último, si proponían acciones adecuadas para mejorar o ampliar el proceso a partir de las interpretaciones realizadas.

En este análisis se generaron cuatro niveles progresivos de desarrollo de la mirada profesional en EPM:

- $\quad$ Nivel 0, si el EPM no identifica los elementos matemáticos.

- Nivel 1, si identifica los elementos pero no los usa para interpretar la comprensión de los alumnos.

- $\quad$ Nivel 2, si identifica los elementos y los usa para interpretar la comprensión de los alumnos.

- $\quad$ Nivel 3, si además, a partir de las interpretaciones realizadas, propone acciones para mejorar o ampliar el proceso.

Todos los EPM han identificado los elementos matemáticos en las respuestas de los tres alumnos de Primaria, la mayoría los ha utilizado para interpretar su comprensión, pero pocos han sido capaces de proponer acciones de mejora o ampliación. Estos resultados se han traducido cuantitativamente en la asignación de niveles: ningún EPM ha sido asignado al nivel 0 , tres han sido asignados al nivel 1, doce al nivel 2 y cinco al nivel 3.

Los resultados obtenidos han permitido confirmar el carácter progresivo de las tres 
destrezas de la mirada profesional: los EPM que han propuesto acciones adecuadas para algún alumno, previamente habían interpretado su comprensión y los EPM que habían interpretado la comprensión de algún alumno, previamente habían identificado elementos matemáticos significativos en sus respuestas.

Este carácter progresivo confirma las observaciones de Jacobs, Lamb y Philipp (2010) que sostienen que las tres destrezas están anidadas, que para decidir acciones basadas en el pensamiento matemático de los alumnos es conveniente interpretar primero su comprensión y que estas interpretaciones se apoyan en la identificación de los elementos matemáticos significativos en las respuestas de los estudiantes.

El que los veinte EPM participantes identificaran los elementos matemáticos significativos y no todos fueran capaces de utilizarlos para interpretar la comprensión de los alumnos de Educación Primaria, es una evidencia empírica de los diferentes niveles de complejidad cognitiva asociados a cada destreza (LLINARES, 2013) y confirma que identificar los elementos matemáticos significativos en la generalización de patrones tiene menor demanda cognitiva para los EPM que interpretar el pensamiento de los alumnos, que exige un conocimiento más profundo de su pensamiento matemático; interpretar la comprensión de un alumno implica observar las dificultades y los aspectos significativos en sus respuestas, por lo que la interpretación depende de la identificación de los elementos matemáticos significativos (FERNÁNDEZ; VALLS; LLINARES, 2011; JACOBS; LAMB; PHILIPP, 2010; MOUHAYAR; JURDAK, 2012; CALLEJO; ZAPATERA, 2017).

Pocos EPM fueron capaces de proponer acciones adecuadas para mejorar o ampliar el proceso de enseñanza-aprendizaje de los alumnos de Primaria, lo que muestra la dificultad de los EPM para tomar decisiones que se apoyen en la comprensión de los alumnos para mejorar o ampliar el proceso de enseñanza-aprendizaje. Este resultado coincide con los obtenidos por Sánchez-Matamoros, Fernández y Llinares (2014), que señalan que la toma de decisiones presenta una mayor dificultad que identificar e interpretar la manera en la que los estudiantes resuelven los problemas. Esta dificultad aumenta al proponer acciones para ampliar el conocimiento del alumno $\mathrm{C}$, que tiene superados los aspectos cognitivos del proceso de generalización; esta dificultad puede atribuirse a un escaso conocimiento de los EPM sobre la trayectoria de la generalización a lo largo de las diversas etapas educativas como sugiere Zapatera (2018).

De esta manera, el carácter progresivo de las destrezas y de los niveles generados puede servir de base para inferir una trayectoria de aprendizaje de la mirada profesional y ofrecer información a los formadores para interpretar el progreso de los EPM en el desarrollo 
de la mirada profesional en el contexto de la generalización de patrones.

También se ha comprobado una vinculación entre las destrezas de la mirada profesional y las dimensiones de la enseñanza de las matemáticas: la identificación de elementos matemáticos puede vincularse con la dimensión matemática, mientras que la interpretación de la comprensión de los alumnos puede vincularse con la dimensión cognitiva y la propuesta de acciones de mejora y ampliación con la dimensión didáctica, como apuntan Sánchez-Matamoros et al. (2013), Zapatera y Callejo (2013) y Callejo y Zapatera (2017).

Los niveles establecidos y sus descriptores pueden ser el punto de partida para establecer una futura trayectoria de aprendizaje de la mirada profesional en estudiantes para maestro (EPM) y contribuir en el desarrollo de esta competencia profesional, proporcionando referencias para elaborar módulos de enseñanza y ofreciendo información a los formadores para interpretar el progreso de los EPM en el desarrollo de la competencia mirar profesionalmente el pensamiento matemático de los alumnos en el contexto de la generalización de patrones.

\section{Referencias}

ARTIGUE, M. Ingeniería didáctica. En: ARTIGUE, M., DOUADY, R., MORENO, L., GÓMEZ, P. (Ed.). Ingeniería didáctica en educación matemática. Bogotá: Grupo Editorial Iberoamérica, 1995. p. 33-59.

BARNHART, T.; VAN ES, E. Studying teacher noticing: Examining the relationship among preservice science teachers' ability to attend, analyze and respond to student thinking. Teaching and Teacher Education, Amsterdam, v. 45, p. 83-93, jan. 2015.

BARTELL, T. G.; WEBEL, C.; BOWEN, B.; DYSON, N. Prospective teacher learning: recognizing evidence of conceptual understanding. Journal of Mathematics Teacher Education, Norwell, v. 16, p. 57-79, 2013.

CALLEJO, M.L.; ZAPATERA, A. Prospective primary teachers' noticing of students' understanding of pattern generalization. Journal of Mathematics Teacher Education, Norwell, 20 (4), p. 309-333, 2017

CARPENTER, T. P.; FRANKE, M. L.; LEVI, L. Thinking mathematically: integrating arithmetic and algebra in elementary school. Portsmouth: Heinemann, 2003.

CARRAHER, D. W.; MARTINEZ, M. V.; SCHLIEMANN, A. D. Early algebra and mathematical generalization. ZDM Mathematics Education, Karlsruhe, v. 40, p. 3-22, 2008.

CLEMENTS, D.; SARAMA, J. Learning trajectories in mathematics education. Mathematical Thinking and Learning, Brisbane, 6 (2), p. 81-89, 2004.

DREYFUS, T. Advanced mathematical thinking process. Mathematics Education Library, Dordrecht, v. 11, p. 25-41, 1991.

DUBINSKY, E. Reflective abstraction in advanced mathematical Thinking. En: TALL, D. (Ed.). 
Advanced mathematical thinking. Dordrecht: Kluwer Academic Publishers, 1991. p. 95-123.

FERNÁNDEZ, C.; VALLS, J.; LLINARES, S. El desarrollo de un esquema para caracterizar la competencia docente mirar con sentido el pensamiento matemático de los estudiantes. En: MARÍN, M.; FERNÁNDEZ, G.; BLANCO, L.; PALAREA, M. (Ed.). Investigación en Educación Matemática XV. Ciudad Real: SEIEM, 2011. p. 351-360.

HAREL, G.; TALL, D. The general, the abstract, and the generic in advanced mathematics. For the Learning of Mathematics, New Westminster, 11 (1), p. 38-42, 1991.

JACOBS, V. R.; LAMB. L. C.; PHILIPP, R. A. Professional noticing of children's mathematical thinking. Journal for Research in Mathematics Education, Reston, 41 (2), p. 169-202, 2010.

LLINARES, S. Professional Noticing: A component of the mathematics teacher's professional practice. Sisyphus, Journal of Education, Lisboa, 1 (3), p. 76-93, 2013.

MASON, J. Researching your own practice: The discipline of noticing. London: Routledge Falmer, 2002.

MAGIERA, M. T.; VAN DEN KIEBOOM, L. A.; MOYER, J. C. An exploratory study of pre-service middle school teachers' knowledge of algebraic thinking. Educational Studies in Mathematics, Dordrecht, 84(1), p. 93-113. 2013.

MOUHAYAR, R. R.; JURDAK, M. E. Teachers' ability to identify and explain students' actions in near and far figural pattern generalization tasks. Educational Studies in Mathematics, Dordrecht, v. 82, p. 379-396, 2012.

PÓLYA, G. Patterns of Plausible Inference. Princeton: Princeton University Press, 1954.

RADFORD, L. Iconicity and contraction: a semiotic investigation of forms of algebraic generalizations of patterns in different contexts. The International Journal on Mathematics Education (ZDM), Karlsruhe, 40(1), p. 83-96, 2008.

RADFORD, L. The progressive development of early embodied algebraic thinking. Mathematics Education Research Journal, Dordrecht, v. 26, p. 257-277, 2014.

RIVERA, F. D. Second grade students' preinstructional competence in patterning activity. En: PINTO, M. F.; KAWASAKI, T. F. (Ed.). Proceedings of the 34th Conference of the International Group for the Psychology of Mathematics Education. Belo Horizonte: PME, 2010, v. 4, p. 81-88.

SÁNCHEZ-MATAMOROS, G.; FERNÁNDEZ, C.; LLINARES, S. Developing pre-service teachers' noticing of students' understanding of the derívate concept. International Journal of Science and Mathematics Education, Taipei, 13 (6), p. 1305-1329, 2014.

SÁNCHEZ-MATAMOROS, G.; FERNÁNDEZ, C.; LLINARES, S.; VALLS, J. El desarrollo de la competencia de estudiantes para profesor de matemáticas de educación secundaria en identificar la comprensión de la derivada en estudiantes de bachillerato. En BERCIANO, A.; GUTIÉRREZ, G.; ESTEPA, A.; CLIMENT, N. (Ed.). Investigación en Educación Matemática XVII. Bilbao: SEIEM, 2013, p. $501-509$.

SCHACK E. O.; FISHER M. H.; THOMAS J. N.; EISENHARDT S.; TASSELL J.; YODER M. Prospective elementary school teachers' professional noticing of children's esarly numeracy. Journal of Mathematics Teacher Education, Norwell, v. 16, p. 379-397, 2013.

SHERIN, M. G.; JACOBS, V. R.; PHILIPP, R. A. (Ed.). Mathematics teacher noticing: Seeing 
through teachers' eyes. New York: Routledge, 2011.

SHERIN, M. G.; VAN ES, E. A. Using video to support teachers' ability to notice classroom interactions. Journal of Technology and Teacher Education, Waynesville, v. 13, p. 475-491, 2005.

SIMON, M. A. Reconstructing mathematics pedagogy from a constructivist perspective. Journal for Research in Mathematics Education, Reston, v. 26, p. 114-145, 1995.

STEFFE, L. P. Learning stages in the construction of the number sequence. En: BIDEAUD, J.; MELJAC, C.; FISCHER, J. (Ed.). Pathways to number: Children's developing numerical abilities. Hillsdale: Lawrence Erlbaum, 1992. p. 83-88.

STRAUSS, A.; CORBIN, J. Grounded theory methodology: An overview. En: DENZIN, N. K.; LINCOLN, Y. S. (Ed.). Handbook of qualitative research. Thousand Oaks: Sage, 1994. p. 273-285. VAN ES, E. A. A framework for learning to notice student thinking. En: SHERIN, M. G.; JACOBS, V.; PHILIPP, R. (Ed.). Mathematics teacher noticing: Seeing through teachers' eyes. New York: Routledge, 2011. p. 134-151.

VAN ES, E. A.; SHERIN, M. G. Learning to Notice: Scaffolding new teachers' interpretations of classroom interactions. Journal of Technology and Teacher Education, Waynesville, 10 (4), p. 571596, 2002.

WARREN, E. Young children's ability to generalize the pattern rule for growing patterns. En: CHICK, H. L.; VINCENT, J. L. (Ed.). Proceedings of the 35th Conference of the International Group for the Psychology of Mathematics Education. Melbourne: PME, 2005, v.1, p. 305-312.

WILSON, P. H.; MOJICA, G. F.; CONFREY, J. Learning trajectories in teacher education: Supporting teachers' understandings of students' mathematical thinking. Journal of Mathematical Behaviour, Amsterdarm, v. 32, p. 103-121, 2013.

ZAPATERA, A. Cómo alumnos de educación primaria resuelven problemas de generalización de patrones. Una trayectoria de aprendizaje. Revista latinoamericana de investigación en matemática educativa, México D.F., 21 (1), p. 87-114, 2018.

ZAPATERA, A. Como interpretan los estudiantes para maestro el pensamiento algebraico de los alumnos de primaria. Uno. Revista de Didáctica de las Matemáticas, Barcelona, v. 68, p. 30-38, $2015 \mathrm{a}$.

ZAPATERA, A. La competencia mirar con sentido de estudiantes para maestro (EPM) analizando el proceso de generalización en alumnos de Educación Primaria. Tesis doctoral. Universidad de Alicante, $2015 b$.

ZAPATERA, A.; CALLEJO, M.L. El conocimiento matemático y la mirada profesional de estudiantes para maestro en el contexto de la generalización de patrones. Caracterización de perfiles. Revista complutense de educación, Madrid, 29 (4), p. 1217-1235, 2018.

ZAPATERA, A.; CALLEJO, M.L. Preservice primary teacher's noticing of students' generalization process. En LINDMEIER, A.M.; HEINZE, A. (Ed.). Proceedings of the 37th Conference of the International Group for the Psychology of Mathematics Education. Kiel: PME, 2013, v.4, p. 425432. 